\title{
SPEEDING UP NON-GAUSSIAN POLSAR IMAGE ANALYSIS
}

\author{
A.P. Doulgeris* \\ UiT The Arctic University of Norway \\ Department of Physics and Technology \\ Troms $\varnothing$, Norway
}

\author{
D. $H u^{\dagger}$ \\ The Institute of Electronics \\ Chinese Academy of Sciences (IECAS) \\ Beijing, China
}

\begin{abstract}
Non-Gaussian statistical models fit SAR data better than Gaussian-based statistics, in most cases, but are complicated and time-consuming to use for unsupervised image segmentation via probabilistic clustering. The more advanced the model, the more complicated and slow the clustering. The U-distribution has been demonstrated to be one of the most flexible models, capturing the Gaussian/Wishart, the K-distribution and the $G_{0}$ models as special cases, but can take hours or days to process a full sized image, depending on the chosen sensitivity. This work explains some computational improvements that drastically reduces the processing time, whilst maintaining the segmented results. The efficiencies are obtained by some smart re-parameterisations of the distribution model parameters allowing the use of lookup-tables for the density function evaluation and parameter estimation. Real images and computation times are shown as a demonstration.
\end{abstract}

Index Terms - Synthetic aperture radar, non-Gaussian modelling, clustering, image processing, look-up-tables

\section{INTRODUCTION}

Non-Gaussian modelling for synthetic aperture radar images has demonstrated advantages to a strictly Gaussian-based analysis and can be used for supervised image classification given training data, or in its unsupervised form for unlabelled images segmentation, which is the focus of this paper. Segmentation via clustering involves assigning each pixel in an image to one of several groups based upon probabilistic similarity. The end result, a segmentation, is an image with a finite number of statistically distinct clusters, which presumably represent real world classes, but the identity of these clusters is unknown. Additional information, like training data or decision criteria, would be required to determine and assign the class labels and thereby turn the segmentation into

\footnotetext{
*With support of the Norwegian Research Council funded Centre for Integrated Remote Sensing and Forecasting for Arctic Operations (CIRFA) under project number 237906.

${ }^{\dagger}$ Supported by the National Natural Science Foundation of China (NSFC) under Grant 61331017
}

a true classification. Nevertheless, an unlabelled segmentation is already valuable for some applications of a more exploratory nature.

Statistical distinction is usually determined by applying the expectation maximisation algorithm with an assumed parametric model to determine the unknown cluster parameters and assign the pixels to their most likely cluster. The resulting clusters are strongly influenced by the number of clusters and the choice of probability density model, and clearly a well fitting model should produce more satisfactory results. To this end, various models have been applied to SAR and PolSAR data, from the un-textured (homogeneous) Wishart model [1,2], to the well known $K$-distribution [3, 4] and $G_{0}$ distribution [5, 6] for heterogeneous data with one texture parameter each, and to the doubly flexible $U$-distribution $[7,8]$ and $G$-distribution [9] models with two texture parameters. The more flexible models were generally shown to be better fitting to some real world classes as well as having some theoretical reasoning for fine scale class mixtures sweeping around in the texture space. The log-cumulant diagrams, showing second and third order log-cumulants of the distributions, clearly visualises the shape space and flexibility of these models, see Fig. 1.

However, the advantages of greater flexibility come at the cost of greater complexity. There are more texture parameters to estimate and more complicated mathematical equations to evaluate, and this is for every iteration, and for every cluster,

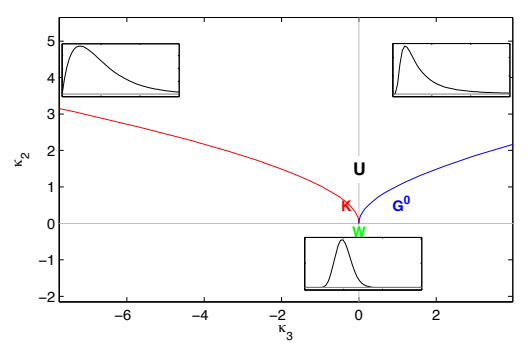

Fig. 1. Log-cumulant shape-space, indicating general intensity profiles for 32-look data, the Wishart point, the $K$ and $G_{0}$ lines, and the U-distribution covers the area in between. 
and for every pixel in the image. For large satellite PolSAR images, which can comprise of many millions of pixels, such non-Gaussian clustering can be a rather time-consuming process. The long processing time is probably the main reason that these non-Gaussian clustering models have not attained more widespread use, since they have been generally demonstrated to have measurably better segmentation results.

This paper describes some new ideas to re-parameterise the equations to produce smoothly varying functions that are amenable to low order approximation and fast evaluation with look-up-tables (LUT), and goes beyond those mentioned previously $[10,11]$. Firstly, the U-distribution expression is shown to be smoothly varying with respect to a compound observable term and may be approximated with linear or cubic spline interpolation for use in a look-up-table. This sped up the repeated probability evaluation part of the code. Secondly, the parameter estimation was dramatically reduced by avoiding the minimum distance search for shape parameters and using a LUT directly on the observed log-cumulants. Since the significance of the texture parameters varies nonlinearly in log-cumulant space, the look-up grid was chosen at logarithmic radial spacing fanning out in the shape space. We demonstrate manyfold speed improvements with these improvements in the U-distribution density function evaluation and parameter estimation. Comparison is made to the previous (slow) algorithm, by the total processing time and by visual observation of the segmented images.

We have not considered parallel processing methods here, because multiple CPUs will only gain a few-fold speed-up for some parts of the code and most GPUs cannot perform many of the more complicated functions, like the Kummer-U hypergeometric function. In contrast, the LUT approach can use the high-level processing and still gain significant gains with little loss of accuracy, and, once the LUT has been developed and proven, the whole interpolation approach can be taken to the GPU in a very trivial way.

\section{METHODOLOGY}

The starting point for this work is the previous clustering algorithm [8] that fits a mixture of U-distribution models to the data, automatically determines an appropriate number of clusters and all class parameters, and returns an unsupervised image segmentation image. It is based upon a modified expectation maximisation algorithm that includes an extra goodnessof-fit testing loop to determine the number of clusters.

The $U$-distribution is defined by the probability density function of the observable covariance matrix $\mathbf{C}$ with five class parameters as

$$
\begin{aligned}
p_{\mathbf{C}}(\mathbf{C} ; d, L, \boldsymbol{\Sigma}, \alpha, \lambda)=\frac{L^{L d}}{\Gamma_{d}(L)} \frac{|\mathbf{C}|^{L-d}}{|\boldsymbol{\Sigma}|^{L}}\left(\frac{\alpha}{\lambda-1}\right)^{L d} \\
\times U\left(L d+\lambda, L d-\alpha+1, \frac{L \alpha}{\lambda-1} \operatorname{tr}\left(\boldsymbol{\Sigma}^{-1} \mathbf{C}\right)\right.
\end{aligned}
$$

where $d$ is the polarimetric dimension, $L$ is the number of looks (or equivalent number of looks), $\boldsymbol{\Sigma}$ is the class mean covariance matrix, and $\alpha$ and $\lambda$ are the shape parameters. $\Gamma_{d}(\cdot)$ denotes the multivariate gamma function of the complex kind, defined as

$$
\Gamma_{d}(s)=\pi^{\frac{d(d-1)}{2}} \Pi_{i=0}^{d-1} \Gamma(s-i)
$$

with $\Gamma(\cdot)$ being the ordinary Euler gamma function, and $U(\cdot, \cdot, \cdot)$ is the Kummer $\mathrm{U}$ function, defined as

$$
U(a, b, z)=\frac{1}{\Gamma(a)} \int_{0}^{\infty} e^{z u} u^{a-1}(1+u)^{b-a-1} \mathrm{~d} u
$$

The original algorithm used for the Kummer-U function internally performs numerical integration as in (2), either in mathematical libraries or by explicitly performing said integration in MatLab code. This function evaluation takes a significant proportion of the overall processing time and is ripe for optimisation. The Kummer-U term in (1), and in fact the whole U-distribution probability density function (PDF), depends upon the global parameters $d$ and $L$, the class specific texture parameters $\{\alpha, \lambda\}$, as well as the class mean covariance $\boldsymbol{\Sigma}$ and the observable $\mathbf{C}$ through the combined term $\operatorname{tr}\left(\boldsymbol{\Sigma}^{-1} \mathbf{C}\right)$. Given that the texture terms are non-linearly related to shape variation and asymptotically approach infinity for the (Gassian-based) Wishart case and that the trace term is essentially a geometric ratio, then it is unsurprising that the probability density function values are more smoothly varying when plotted as $\log$-U-distribution versus $\log (\alpha), \log (\lambda)$ and $\log \left(\operatorname{tr}\left(\boldsymbol{\Sigma}^{-1} \mathbf{C}\right)\right)$, as shown in figure 2 .

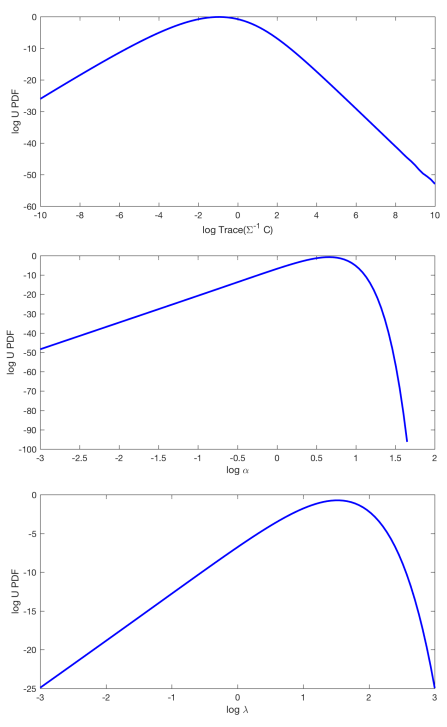

Fig. 2. Example of logarithmic U distribution PDF changing with $\log \left(\operatorname{tr}\left(\boldsymbol{\Sigma}^{-1} \mathbf{C}\right)\right), \log (\alpha)$, and $\log (\lambda)$, respectively. The fixed parameters in each plot are $\alpha=5, \lambda=5$, and $\mathbf{C}=\mathbf{\Sigma}$. 
The smoothly varying function could now be approximated by a linear or cubic spline LUT with good results and with significantly faster evaluation time than numerical integration. However, the overall computation time was still lengthy and we realised that the U-distribution PDF evaluation was only part of the problem and the next processing bottleneck was for the texture parameter estimation.

Each cluster (class) is defined by its own mean covariance matrix $\boldsymbol{\Sigma}$, and the two texture parameters $\alpha$ and $\lambda$. In the existing algorithms, the parameter $\boldsymbol{\Sigma}$ is determined with the sample covariance matrix estimator, which becomes a membership weighted mean over the $\mathbf{C}$ matrix data samples in the fuzzy clustering sense and is reasonably efficient. The method for estimating the two texture parameters, $\alpha$ and $\lambda$, however, is much more cumbersome, because there are no close-form maximum likelihood solutions. Texture parameter estimation is achieved with the method of matrix log-cumulants.

The method of matrix log-cumulants states that the logcumulants of order 2 or higher are independent of the mean matrix parameter and only dependent upon the shape parameters. For this work we utilise log-cumulants $\kappa_{2}$ and $\kappa_{3}$, from the theoretical population log-cumulant expression

$\kappa_{\nu>1}(L, \alpha, \lambda)=\psi_{d}^{(\nu-1)}(L)+d^{\nu}\left(\psi^{(\nu-1)}(\alpha)-\psi^{(\nu-1)}(\lambda)\right)$

where $\psi_{d}^{(\nu)}(\cdot)$ is the multivariate polygamma function defined as

$$
\psi_{d}^{(\nu)}(L)=\sum_{i=0}^{d-1} \psi^{(\nu)}(L-i)
$$

and $\psi^{(\nu)}(\cdot)$ is the usual polygamma function of order $\nu$. Such expressions for the log-cumulants do not allow for direct inversion to obtain the maximum likelihood parameter estimates from sample log-cumulants and, hence, a minimum distance search is usually employed and which leads to the processing bottleneck. These parameters are only obtained for the subsequent re-substitution into the PDF evaluation to calculate likelihoods, and hence this whole estimation process may be avoided by creating a LUT directly on the observed $\log$-cumulant values. Either getting $\alpha$ and $\lambda$ from LUTs and using the original U-distrtibution PDF parameterisation, or more efficiently by directly looking-up the U-distrtibution PDF evaluation with respect to observed log-cumulants, essentially skipping the intermediate parameter evaluation.

A look-up-table may be created with a grid of $\kappa_{2}, \kappa_{3}$ log-cumulant space to efficiently determine the corresponding nearest $\alpha, \lambda$ parameters and lead to a much faster evaluation. Considering the asymptotic relations and fan-like manifold of the log-cumulant with regards to the texture parameters, we choose to use a grid based upon radial distance from the Wishart point and angle around the hemisphere to neatly reduce our gridded range. In addition, the distance is sampled logarithmically to increase the detail approaching the Wishart point. An example grid is shown in figure 3 .

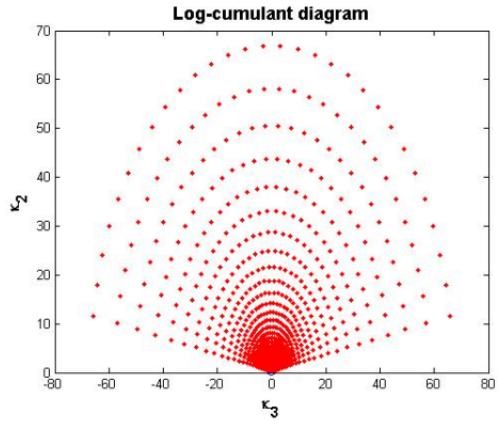

Fig. 3. An example group of LUT nodes plot on the $\kappa_{2}, \kappa_{3}$ plane. The red dots represent the LUT nodes.

In summary, the new clustering strategy is to determine the equivalent number of looks using an unsupervised estimation procedure in a pre-processing stage [12], and then prebuild a LUT for the U-distribution PDF evaluation. Subsequently determine each class mean covariance matrix (with a membership weighted average over the samples) and the sample matrix log-cumulants of order 2 and 3 (also using membership weighted log-cumulant sums) ready for probability evaluation via the LUT within the modified expectation maximisation algorithm. The number of clusters is determined by the goodness-of-fit testing loop and finished with the optional Markov random field contextual smoothing.

\section{RESULTS}

The main results are the segmentation images with the new look-up-table strategy compared to the images without the look-up-table, along with the computational times of the processing. These results show the suggested parameter estimation replacement with a LUT, but only a partial implementation of the probability evaluation, because only the Kummer$\mathrm{U}$ hypergeometric function is implemented as a LUT, and other power and gamma function factors are still evaluated. We did not get time to implement the entire PDF as a LUT, and hence we could expect to speed up the processing further.

An example for San Francisco is shown in figure 4. The images are visually very similar with only $2.5 \%$ of pixels having a different class label. The times shown, in table 1 , indicate the total clustering time, including various set-up, table-generation, actual clustering, MRF smoothing and screen plotting functions. This image was $240 \times 240$ pixels and larger images may gain relatively more.

The new strategy allows the table to be prebuilt, which can save much more time, as indicated in parentheses in table 1. 
Table 1. Total processing-time comparison.

\begin{tabular}{|l|c|c|}
\hline & Integrated U & new LUT strategy (prebuilt) \\
\hline time [s.] & 410 & $26.1(14.7)$ \\
\hline \multicolumn{2}{|l|}{ speed-up from integrated } & $\times 15.7(\times 27.9)$ \\
\hline
\end{tabular}

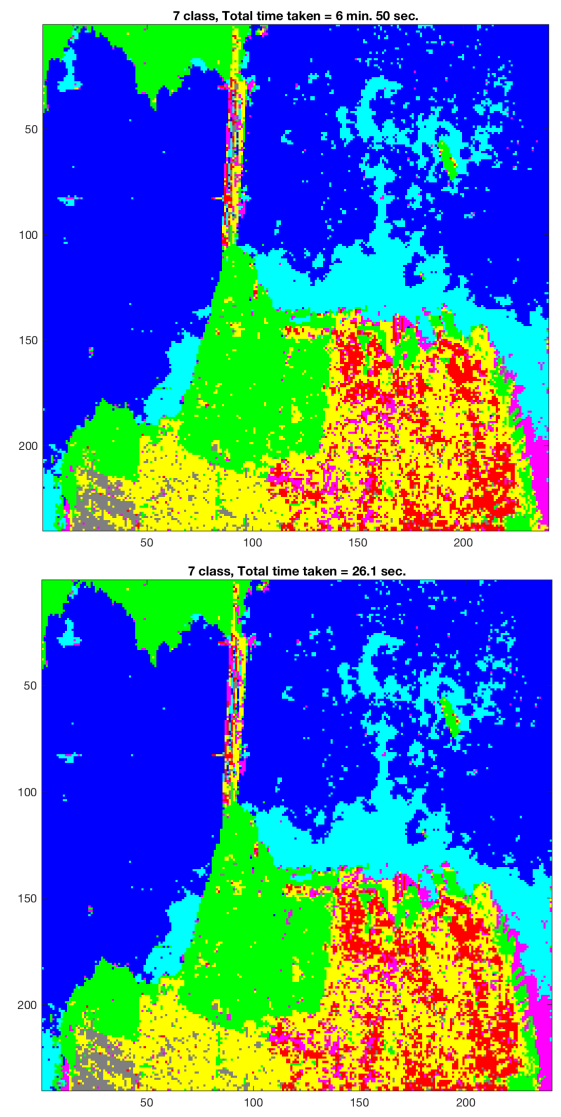

Fig. 4. Preliminary figures from algorithm, without LUT and with LUT. Only $2.5 \%$ of pixels have changed labels.

\section{CONCLUSION}

The look-up-table strategy with these smooth parameterisations allows for manyfold computation time speed-up without a significant effect on the image results. The speed improvements now make it comparable in speed to a normal (Gaussian-based) Wishart analysis, which may open up more opportunity to benefit from the non-Gaussian model fitting.

\section{REFERENCES}

[1] J.S. Lee, M.R. Grunes, and R. Kwok, "Classification of multi-look polarimetric SAR imagery based on the complex Wishart distribution," in Int. J. Remote Sensing, November 1994, vol. 15.
[2] Jong-Sen Lee, Mitchell R. Grunes, Thomas L. Ainsworth, Dale L. Schuler, and Shane R. Cloude, "Unsupervised classification using polarimetric decomposition and the complex Wishart distribution," IEEE Trans. Geosci. Remote Sens., vol. 37, no. 5, pp. 2249-2259, 1999.

[3] J. S. Lee, D. L. Schuler, R. H. Lang, and K. J. Ranson, "K-Distribution for multi-look processed polarimetric SAR imagery," in IEEE Int. Geosci. Remote Sensing Symp., Pasedena, USA, August 1994, vol. 4, pp. 21792181.

[4] A. P. Doulgeris, S. N. Anfinsen, and T. Eltoft, "Automated non-Gaussian clustering of polarimetric synthetic aperture radar images," IEEE Trans. Geoscience and Remote Sensing, vol. 49, no. 10, pp. 3665-3676, 2011.

[5] A.C. Frery, H.-J. Muller, C.C.F. Yanasse, and S.J.S. Sant'Anna, "A model for extremely heterogeneous clutter," Geoscience and Remote Sensing, IEEE Transactions on, vol. 35, no. 3, pp. 648 -659, May 1997.

[6] C. C. Freitas, A. C. Frery, and A. H. Correia, "The polarimetric G distribution for SAR data analysis," Environmetrics, vol. 16, no. 1, pp. 13-31, 2005.

[7] L. Bombrun and J.-M. Beaulieu, "Fisher distribution for texture modeling of polarimetric sar data," Geoscience and Remote Sensing Letters, IEEE, vol. 5, no. 3, pp. 512 -516 , July 2008.

[8] A. P. Doulgeris, "An automatic u-distribution and markov random field segmentation algorithm for PolSAR images," Transactions on Geoscience and Remote Sensing, vol. 53, no. 4, pp. 1819-1827, 2015.

[9] S. Khan and R. Guida, "On single-look multivariate cal G distribution for polsar data," IEEE Journal of Selected Topics in Applied Earth Observations and Remote Sensing, vol. 5, no. 4, pp. 1149-1163, Aug 2012.

[10] D. Hu, A. P. Doulgeris, X. Qiu, and B. Lei, "A fast automatic u-distribution segmentation algorithm for polsar images," in 2016 IEEE International Geoscience and Remote Sensing Symposium (IGARSS), July 2016, pp. 6336-6339.

[11] Dingsheng Hu, Anthony Paul Doulgeris, Xiaolan Qiu, Bin Lei, and Wei Zhang, "A fast automatic udistribution segmentation algorithm for polsar images," Remote Sensing Letters, 2017 (Submitted).

[12] D. Hu, S. N. Anfinsen, X. Qiu, A.P. Doulgeris, and B. Lei, "Unsupervised mixture- eliminating estimation of equivalent number of looks for polsar data," IEEE Transac-tions on Geoscience and Remote Sensing, 2016. 